\title{
Detection of high-energy compounds using photoluminescent silicon nanocrystal paper based sensors $\uparrow$
}

Received 26th November 2013 Accepted 8th January 2014

\author{
Christina M. Gonzalez, ${ }^{a}$ Muhammad lqbal, ${ }^{a}$ Mita Dasog, ${ }^{a}$ Davin G. Piercey, ${ }^{c}$ \\ Ross Lockwood, ${ }^{d}$ Thomas M. Klapötke ${ }^{c}$ and Jonathan G. C. Veinot ${ }^{\star a b}$
}

DOI: $10.1039 / c 3 n r 06271 f$

www.rsc.org/nanoscale

Luminescent silicon nanocrystals (Si-NCs) surface functionalized with dodecyl groups were exposed to solutions of nitroaromatic compounds including nitrobenzene, nitrotoluene, and dinitrotoluene. It was found that Si-NC luminescence was quenched upon exposure to nitroaromatics via an electron transfer mechanism as indicated by Stern-Volmer analysis. This quenching was exploited and a straightforward paper-based Si-NC sensor was developed. This paper motif was found to be sensitive to solution, vapor, and solid phase nitroaromatics, as well as solution borne RDX and PETN.

\section{Introduction}

Of late, sensing high energy materials (i.e., explosives) has received substantial attention because of its obvious importance to security and forensics; detection of these materials is also crucial because many are toxic and pose environmental risks. ${ }^{6,7,9}$ Modern methods for detecting explosives include gas chromatography coupled with mass spectrometry, ion mobility spectrometry, surface enhanced Raman spectroscopy, and energy dispersive X-ray spectroscopy. ${ }^{10-13}$ Unfortunately, all of these methods are infrastructure intensive and cannot be readily implemented in the field or outside a laboratory setting. ${ }^{14}$ There is a concerted push to develop sensing technologies that are cost effective. One approach is to use paper substrates as sensing motifs. For example, paper-based surface enhanced Raman spectroscopy substrates for explosive detection have also been developed. ${ }^{31,32}$ In this context, the development of complementary techniques for straightforward, rapid, on-site detection is of paramount importance.

\footnotetext{
${ }^{a}$ Department of Chemistry, University of Alberta, Edmonton, Alberta T6G 2G2, Canada. E-mail: jveinot@ualberta.ca; Fax: +1-780-492-8231; Tel: +1-780-492-7206 ${ }^{b} N R C$ National Institute for Nanotechnology, Edmonton, Alberta, T6G 2M9, Canada 'Department of Chemistry, Energetic Materials Research, Ludwig-Maximilian University, Butenandtstr. 5-13 (D), 81377 München, Germany

${ }^{d}$ Department of Physics, University of Alberta, Edmonton, Alberta T6G 2G2, Canada $\dagger$ Electronic supplementary information (ESI) available: Experimental details, Si-NC characterization, fluorescence spectra, solid residue testing and vapor study images. See DOI: 10.1039/c3nr06271f
}

An attractive approach toward realizing this goal is the development of fluorescent sensors that respond to these compounds. These sensors are usually comparatively simple, require minimal infrastructure, are cost effective, and exhibit adequate sensitivity as well as response times. ${ }^{8}$ Recently, luminescent nanomaterials (e.g., Cd-based quantum dots (QDs)) have been explored as fluorescent sensors because of their exquisite tunability. ${ }^{15,24,25}$ Freeman and coworkers successfully employed fluorescent, functionalized CdSe/ZnS QDs to detect trace quantities of trinitrotoluene (TNT) and cyclotrimethylenetrinitramine (RDX). ${ }^{5}$ In efforts to render these systems portable and increase their compatibility with field applications, researchers interfaced the active nanomaterials with common filter paper to afford a detection system. Zhang and coworkers coated filter paper with dual-emission CdTe QDs that luminesce different colours in the presence of TNT. ${ }^{3}$ Similarly, Ma and coworkers used the molecular emitter 8-hydroxyquinoline aluminum and nanospheres to detect 2,4,6trinitrophenol (TNP). ${ }^{4}$

Quantum dots have the clear advantage over molecule-based emitters that they do not photobleach, however, CdSe and CdTe QDs are toxic. ${ }^{21}$ Furthermore, regulations exist or are pending in numerous jurisdictions that limit their widespread use in industrial and consumer applications - new materials must be explored. ${ }^{21}$ Silicon nanocrystals (Si-NCs) are attractive alternative materials that have all the advantages of Cd-based quantum dots (e.g., tailorability and photostability) with the clear benefit of being non-toxic. Content and coworkers showed that the photoluminescence of hydride terminated porous silicon films was quenched upon exposure to dinitrotoluene (DNT), TNT, and nitrobenzene (NB) vapors. These quenching processes are believed to occur via a reversible electron transfer mechanism or irreversible chemical oxidation depending on the duration of vapor exposure. ${ }^{1}$ However, the hydride terminated porous silicon surface is readily oxidized upon exposure to air and is fragile, thus making it impractical for field applications. Germanenko and coworkers demonstrated that the red luminescence of web-like agglomerated silicon nanocrystals 
( $d \sim 5-6 \mathrm{~nm}$ ) bearing a 1-2 $\mathrm{nm}$ oxide surface layer was quenched when exposed to nitroaromatic compounds. ${ }^{2}$ Unfortunately, the luminescence of these materials was not affected by explosive-related compounds NB and mononitrotoluene (MNT) that are common degradation products of nitro-class explosives typically found in landmines. ${ }^{26}$ These early reports demonstrate the promise of Si-based nanomaterials and suggest that photoluminescent Si-NCs could be used as sensors for nitro-containing explosives. To the best of our knowledge, no study has been reported on employing well-defined freestanding alkyl terminated surface silicon nanocrystals as nitroaromatic, nitoramine or nitrate ester explosive detection systems.

In this work, we describe the detection of a series of nitroaromatic compounds (i.e., mononitrotoluene (MNT), nitrobenzene (NB), dinitrotoluene (DNT), and trinitrotoluene (TNT)), as well as nitramine RDX and nitrate ester PETN by exploiting the optical response of non-toxic dodecyl functionalized Si-NCs in solution. We subsequently extend this investigation and outline the fabrication and application of an air stable fluorescent paper detector based on these particles. This paper-based system showed rapid detection of nitroaomatics, nitroamine, and nitrate esters by luminescence quenching in solution, vapor and solid phases at nanogram levels.

\section{Results and discussion}

\section{Characterization of dodecyl functionalized Si-NCs}

Oxide-embedded Si-NCs were obtained by thermally induced disproportionation of HSQ. ${ }^{19}$ After etching with HF, red-emitting, hydride terminated Si-NCs were obtained and immediately functionalized with dodecene via thermally induced hydrosilylation as reported previously. ${ }^{19}$ Material characterization is summarized in Fig. S1. $\dagger$ The FTIR spectrum (Fig. S1A $\dagger$ ) shows features characteristic of alkyl terminated surfaces at $2920 \mathrm{~cm}^{-1}$ (C-H stretching) and $1450 \mathrm{~cm}^{-1}(-\mathrm{C}-\mathrm{H}$ bending) consistent with dodecyl functionalization. ${ }^{20}$ Features observed at $2110 \mathrm{~cm}^{-1}$ and $1050 \mathrm{~cm}^{-1}$ indicate that small amounts of $\mathrm{SiH}_{x}$ and $\mathrm{Si}-\mathrm{O}-\mathrm{Si}$ functionalities, respectively, remain following alkyl modification. The PL spectrum of the dodecyl functionalized SiNCs in toluene (Fig. S1B $\dagger$ ) shows a peak intensity maximum at $643 \mathrm{~nm}$. The morphology of the functionalized Si-NCs was evaluated using transmission electron microscopy (Fig. S1C $\dagger$ ) which indicates that the particles are pseudospherical with an average diameter of $3.7 \pm 0.4 \mathrm{~nm}$ (Fig. S1D $\dagger$ ).

\section{Fluorescence quenching by nitroaromatic compounds}

Upon addition of nitroaromatic compounds (i.e., NB, MNT, and DNT) to solutions of dodecyl functionalized Si-NCs, luminescence was quenched. Fig. 1A shows titration curves of the fluorescence peak intensity of toluene solutions containing $1 \mathrm{mg} \mathrm{mL}{ }^{-1} \mathrm{Si}-\mathrm{NCs}$ as a function of DNT concentrations ranging from 0.05 to $25 \mathrm{mM}$. Consistent with previous reports, the degree of Si-NCs PL quenching was proportional to the concentration of DNT (i.e., the higher the DNT concentration, the more efficient the quenching). In addition, no shift in PL
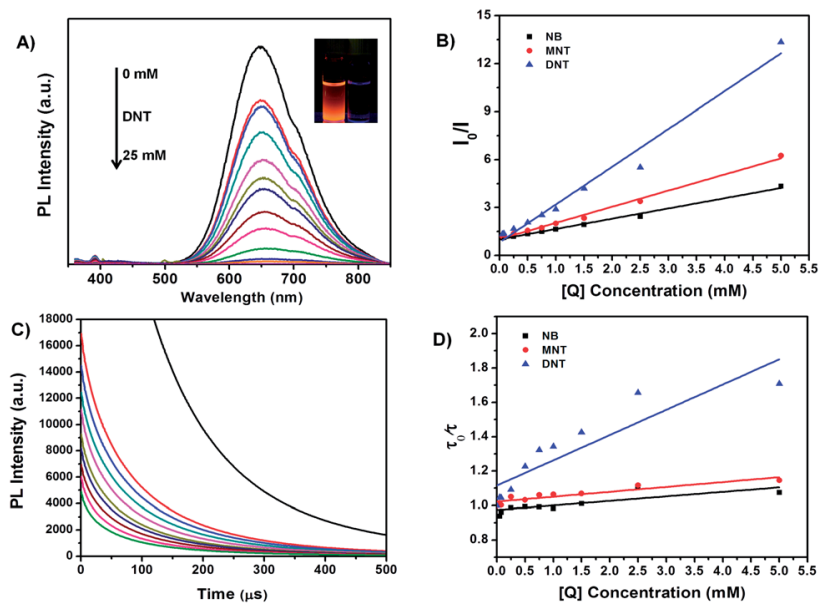

Fig. 1 (A) Fluorescence quenching spectra of Si-NCs by increasing concentrations of DNT in solution with an inset showing the quenching effect with 0 and 25 mM DNT atop bench-top UV-lamp. (B and $\mathrm{D}$ ) the Stern-Volmer plots for the quenching efficiencies and $\mathrm{PL}$ lifetimes of NB, MNT and DNT at different concentrations. (C) The PL lifetime decays of Si-NCs with increasing concentrations of DNT. The quencher concentrations for all studies can be found in Table S1. $\dagger$

maximum or changes in the line shape of the PL spectrum was obtained. Similar behaviour is observed in the titration curves for the NB and MNT compounds investigated (Fig. S2A and $\mathrm{B} \dagger$ ).

To gain a more complete understanding of the quenching behaviour induced by NB, MNT, and DNT on dodecyl functionalized Si-NCs, PL data were evaluated using the SternVolmer equation:

$$
I_{0} / I=K_{\mathrm{sv}}[\mathrm{Q}]+1
$$

where, $I_{0}$ and $I$ are the fluorescence intensity in the absence and presence of nitroaromatic compounds, respectively. [Q] is the nitroaromatic compound concentration, and $K_{\mathrm{sv}}$ is the fluorescence quenching constant. Fig. 1B shows a linear relationship of $I_{0} / I$ vs. nitroaromatic compound (NB, MNT, DNT) concentration. In the range of $0.05-5 \mathrm{mM}, \mathrm{NB}, \mathrm{MNT}$, and DNT display linear behaviour indicative of the quenching arising from a dynamic process such as an electron transfer. ${ }^{23}$ It has been proposed, based on the correlation of reduction potential in nitroaromatic compounds, that luminescence quenching of both porous silicon and oxide terminated web-like aggregates of Si-NCs proceeds via an electron transfer pathway., ${ }^{\mathbf{1 , 2 2} 2}$ Suggestions have been made that the electron transfer occurs from the Si nanomaterial conduction band to the vacant $\pi^{*}$ orbital of the nitroaromatic compound, resulting in photoluminescence quenching. ${ }^{2,27}$ If this is the case for the present systems, considering the known reduction potentials of $\mathrm{NB}$, MNT, and DNT (i.e., $-1.15 \mathrm{~V},-1.19 \mathrm{~V}$, and $-0.9 \mathrm{~V} v s$. NHE in acetonitrile, respectively), ${ }^{\mathbf{1 , 2 2}}$ the PL quenching efficiency should decrease with redox potential. As such, DNT should be the most efficient quencher of the three tested here. The $K_{\mathrm{sv}}$ values determined from the analysis presented in Fig. 1B are $6.44(\mathrm{mM})^{-1}, 1.01(\mathrm{mM})^{-1}$, and $2.36(\mathrm{mM})^{-1}$ for NB, MNT, and DNT, respectively. This trend supports the hypothesis 
that quenching is actually occurring via an electron transfer mechanism.

To further verify whether the quenching mechanism is a dynamic process, the PL lifetime of the Si-NCs as a function of concentration of each of the nitroaromatic quenchers (i.e., NB, MNT, DNT) in the range of $0.05-5 \mathrm{mM}$ was studied. If the PL lifetime is independent of the quencher concentration, the quenching mechanism is static and is governed by the formation of a ground state nanoparticle-analyte complex. ${ }^{30}$ Alternatively, if the quenching process is dynamic there will be a decrease in the lifetime because of additional deactivation pathways (e.g., electron transfer) that will shorten the lifetime. ${ }^{30}$ For the present system, increasing the concentration of the nitroaromatic compound resulted in a decrease of lifetime decays (Fig. 1C, S2C and S2D †). These results were then plotted as $\tau_{0} / \tau$ vs. nitroaromatic compound (NB, MNT, DNT) concentrations where $\tau_{0}$ and $\tau$ are the PL lifetimes in the absence and presence of nitroaromatic compounds, respectively (Fig. 1D). As expected, DNT was the most efficient lifetime quencher of all nitro-compounds tested. These results further support that the quenching mechanism is a dynamic process via electron transfer.

To determine the limit of detection (LOD) for PL quenching that functionalized Si-NCs display for toluene solutions of NB, MNT, and DNT, the PL quenching arising from analyte concentrations of the range $0.05-5 \mathrm{mM}$ was evaluated. The LODs for nitroaromatic compounds in toluene were determined using the $3 \sigma$ IUPAC criteria and were calculated to be 1.54, 0.995, and 0.341 $\mathrm{mM}$ (i.e. 184.6, 136.5, and $62.1 \mathrm{ppm}$, respectively) for NB, MNT, and DNT, respectively. ${ }^{\mathbf{1 6 , 1 7}}$ Unfortunately, solution phase LODs in toluene are not as sensitive as previous reports. ${ }^{28}$ The origin of the decreased LODs is unclear, however, it may result from the influence of the alkyl surface termination and is the subject of ongoing investigations. Fortunately, these solution LODs do not preclude the practical usefulness of this Si-NC detection system, which can be extended to the solid residue and vapor detection (vide infra). Furthermore, it is also reasonable that LODs will be improved by appropriate tailoring of the surface functional groups.

\section{Visual detection using paper supported Si-NCs}

Extending the potential utility of the present Si-NC sensing motif, we prepared the filter paper impregnated with luminescent Si-NCs by dip-coating in a toluene solution of the NCs (Scheme 1).
The resulting paper displayed red-orange photoluminescence characteristic of the Si-NCs upon exposure to a standard handheld UV ( $\lambda=365 \mathrm{~nm}$ ) lamp (Fig. 2). To test the sensitivity of this new detection system, $2 \mu \mathrm{L}$ of stock solutions $(0.25,5$, and $25 \mathrm{mM})$ of NB, MNT, and DNT were spotted onto the prepared paper (Scheme 1). Photographs of the exposed papers are shown in Fig. S3. $\dagger$

All concentrations tested resulted in complete quenching of the area spotted for every compound. The results indicate that measurement using the filter paper is more effective at 0.25 and $5 \mathrm{mM}$ concentrations than solution phase measurement by the fluorometer where a complete quenching at these concentrations was not achieved. There was no visible difference in the quenched spots for all concentrations of NB and MNT. However, DNT displayed a dramatic quenching increase in the area surrounding the initial spot of the compound. As seen in solution, DNT is the most effective of the nitroaromatics tested. To further test the application of the filter paper, $25 \mu \mathrm{L}$ of 0.01 mM solutions of explosives TNT, RDX, and PETN were spotted onto the filter paper, and the fluorescence was rapidly and completely quenched for all compounds (Fig. 2). This study showed that the filter paper is not only sensitive to nitroaromatics, but also to nitroamines and nitrate esters, and points out the scope of such a sensor motif in real-world applications.

The same filter paper detector can also be applied in the detection of chemical residues. Placing known quantities of DNT residues (i.e., 4.5, 18.2 and $91.1 \mathrm{ng}$ ) on cotton swabs and exposing the filter paper to the residue (Experimental section in the ESI $\dagger$ ) indicated that the present system can detect as little as $18 \mathrm{ng}$ of DNT (Fig. 3A).

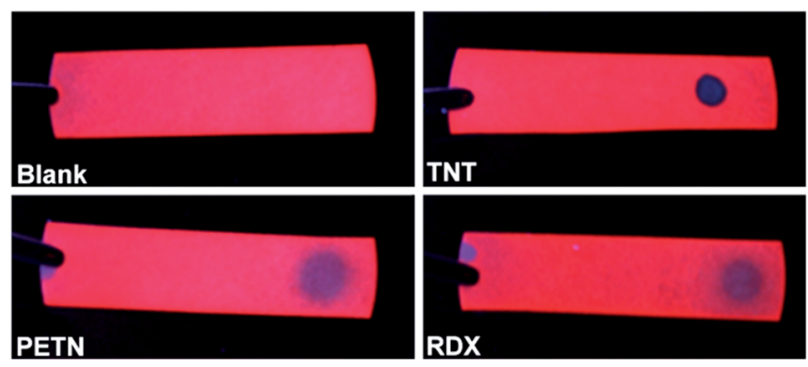

Fig. 2 Images of Si-NC coated filter paper under a handheld UV-lamp without the presence of nitrocompounds and in the presence of solutions of TNT, PETN, and RDX, as indicated.
1.

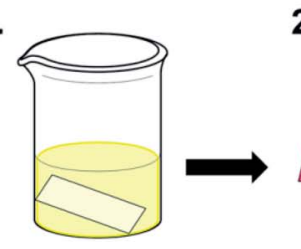

2.

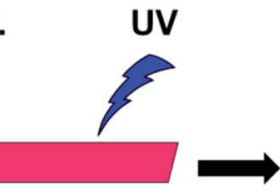

3.

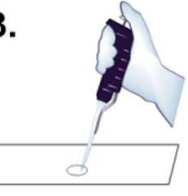

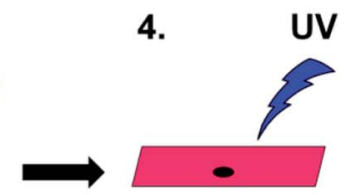

Scheme 1 Schematic representation of the preparation and use of Si-NC based sensor paper. (1) A piece of filter paper is dip coated in a solution of concentrated Si-NCs, (2) the resulting paper is fluorescent under UV light, (3) nitroaromatic solution is spotted onto the sensing paper, and (4) quenching of the spot is observed under UV light. 

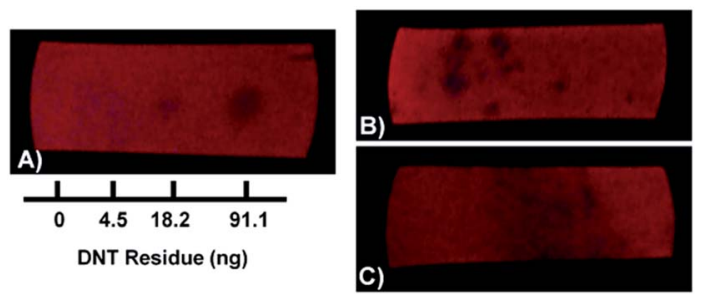

Fig. 3 Solid DNT residue testing onto Si-NC filter paper by (A) cotton swab tips having different amounts of DNT, the DNT residue left after visibly brushing off $0.5 \mathrm{mg}$ DNT from a (B) plastic tray and a (C) cotton fabric, respectively.

The detection of trace residues of DNT on the surfaces of a plastic tray and cotton fabric was also tested (Experimental section in ESI $\dagger$ ). Contact of the exposed surface with the filter detection paper resulted in quenching of the Si-NC luminescence (Fig. 3B and C). Further testing was carried out by wiping a gloved finger that had been previously exposed to solid DNT (Experimental section in ESI $\dagger$ ). Pressing the finger onto the paper successively 4 times resulted in subsequent quenching (Fig. S4 $\dagger$ ). Although the exact quantity of DNT residue on the glove decreased with successive printing, the qualitative signal to noise ratio between the first and last print appears to remain unchanged. ${ }^{29}$ Solid TNT was similarly tested using the glove method, where the contaminated finger was placed on the luminescent area of the detector paper; the luminescence was quenched where the finger was placed (Fig. 4). Of important note, control tests (not shown) with a gloved finger, an ungloved finger, and a finger of someone who recently smoked a cigarette provided no quenching.

To further explore the versatility of the present detection system for nitro-compounds in the vapor phase, the indicator paper was exposed to the headspace above NB (Experimental section in ESI $\dagger$ ). The exposure to NB vapors completely quenched the Si-NCs within 3 minutes (Fig. S5 $\dagger$ ) and quenching was reversed upon exposure to a stream of flowing $\mathrm{N}_{2}$. These results are similar to those reported by Content and coworkers in the previous report using hydride terminated porous silicon, ${ }^{\mathbf{1}}$ and confirm that the present paper motif offers detection of

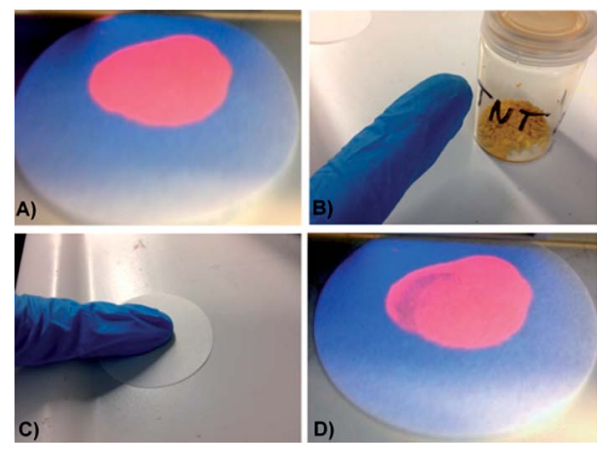

Fig. 4 Images of (A) a spotted filter paper with Si-NCs, (B) a gloved finger with trace amounts of solid TNT, (C) application of solid TNT to the coated filter paper, and (D) observed quenching of luminescent filter paper after contact with the solid TNT residue. nitroaromatics in solution as well as vapor and solid phases. The paper-based system may be best adapted as a reliable frontline screening method for on-site detection where rapid detection of explosives and related compounds could prove useful in areas such as landmines, airport, border security, etc. ${ }^{18}$

\section{Conclusions}

In conclusion, the present study demonstrates that luminescent dodecyl functionalized Si-NCs are quenched in the presence of nitroaromatic, nitroamine, and nitrate ester explosive compounds. When tested in solution, the fluorescence intensity decreased with increasing amount of nitroaromatics. The applicability of these Si-NCs was tested by coating them onto a filter paper to generate a luminescent paper-sensor. The papersensor successfully detects solution, solid and vapor phase nitroaromatic compounds through visualization of fluorescence quenching under a handheld UV lamp. The versatility was further expanded to the sensing of nitroamines and nitrate esters (i.e., RDX and PETN). The method described here offers a non-toxic, portable, rapid, and straightforward sensing system for on-site detection of nitro-groups containing explosives. Further testing and development using different surface functional groups other than dodecene could be used to increase sensitivity and selectivity towards nitroaromatic, nitroamine and nitrate ester explosives or to detect other molecules.

\section{Acknowledgements}

The authors acknowledge funding from the Natural Sciences and Engineering Research Council of Canada (NSERC), Canada Foundation for Innovation (CFI), Alberta Science and Research Investment Program (ASRIP), Alberta Innovates Technology Futures (AITF), and the Department of Chemistry, University of Alberta. We would like to thank W. C. Moffat, M. Skjel, and M. Hoyle for assistance with material characterization. Killam trusts, NSERC and Alberta Innovates (AI) are thanked for studentship to MD. Financial support of this work from the Ludwig-Maximilian University of Munich (LMU), the U.S. Army Research Laboratory (ARL) under grant no. W911NF-09-2-0018, the Armament Research, Development and Engineering Center (ARDEC) under grant no. W911NF-12-1-0467, and the Office of Naval Research (ONR) under grant no. ONR.N00014-10-1-0535 and ONR.N00014-12-1-0538 is gratefully acknowledged. Dr. Al Meldrum is thanked for use of laser facilities for lifetime measurements. All Veinot Team members and Dr. Farooq Wahab are also thanked for useful discussion.

\section{Notes and references}

1 S. Content, W. C. Trogler and M. J. Sailor, Chem. - Eur. J., 2000, 6, 2205.

2 I. N. Germanenko, S. Li and M. S. El-Shall, J. Phys. Chem. B, 2001, 105, 59.

3 K. Zhang, H. Zhou, Q. Mei, S. Wang, G. Guan, R. Liu, J. Zhang and Z. Zhang, J. Am. Chem. Soc., 2011, 133, 8424. 
4 Y. Ma, H. Li, S. Peng and L. Wang, Anal. Chem., 2012, 84, 8415.

5 R. Freeman, T. Finder, L. Bahshi, R. Gill and I. Willner, Adv. Mater., 2012, 24, 6416.

6 B. Balan, C. Vijayakumar, M. Tsuji, A. Saeki and S. Seki, J. Phys. Chem. B, 2012, 116, 10371.

7 M. E. Germain and M. J. Knapp, Chem. Soc. Rev., 2009, 38, 2543.

8 Y. Yang, H. Wang, K. Su, Y. Long, Z. Peng, N. Li and F. Liu, J. Mater. Chem., 2011, 21, 11895.

9 Y. Salinas, R. Martinez-Manez, M. D. Marcos, F. Sancenon, A. N. Costero, M. Parraad and S. S. Gil, Chem. Soc. Rev., 2012, 41, 1261.

10 K. Hakansson, R. V. Coorey, R. A. Zubarev, V. L. Talrose and P. Hakansson, J. Mass Spectrom., 2000, 35, 337.

11 M. Najarro, M. E. D. Morris, M. E. Staymates, R. Fletcher and G. Gillen, Analyst, 2012, 137, 2614.

12 J. M. Sylvia, J. A. Janni, J. D. Klein and K. M. Spencer, Anal. Chem., 2000, 72, 5834.

13 R. D. Luggar, M. J. Farquharson, J. A. Horrocks and R. J. Lacey, X-Ray Spectrom., 1998, 27, 87.

14 J. Feng, Y. Li and M. Yang, Sens. Actuators, B, 2010, 145, 438.

15 R. Tu, B. Liu, Z. Wang, D. Gao, F. Wang, Q. Fang and Z. Zhang, Anal. Chem., 2008, 80, 3458.

16 W. J. Jin, M. T. Fernandez-Arguelles, J. M. Costa-Fernandez, R. Pereiro and R. Sanz-Medel, Chem. Commun., 2005, 883.

17 W. J. Jin, J. M. Costa-Fernandez, R. Pereiro and A. SanzMedel, Anal. Chim. Acta, 2004, 522, 1.
18 G. H. Shi, Z. B. Shang, Y. Wang, W. J. Jin and T. C. Zhang, Spectrochim. Acta, Part A, 2008, 70, 247.

19 C. M. Hessel, E. J. Henderson and J. G. C. Veinot, Chem. Mater., 2006, 18, 6139.

20 J. A. Kelly and J. G. C. Veinot, ACS Nano, 2010, 4, 4645.

21 A. M. Derfus, W. C. W. Chan and S. N. Bhatia, Nano Lett., 2004, 4, 11.

22 J. M. Rehm, G. L. McLendon and P. M. Fauchet, J. Am. Chem. Soc., 1996, 118, 4490.

23 J. C. Scaiano, M. Laferrière, R. E. Galian, V. Maurel and P. Billone, Phys. Status Solidi A, 2006, 203, 1337.

24 J. M. Costa-Fernandez, R. Pereiro and A. Sanz-Medel, TrAC, Trends Anal. Chem., 2006, 25, 207.

25 R. Freeman and I. Wilner, Chem. Soc. Rev., 2012, 41, 4067.

26 J. F. Garcia-Reyes, J. D. Harper, G. A. Salazar, N. A. Charipar, Z. Ouyang and R. G. Cooks, Anal. Chem., 2011, 83, 1084.

27 A. K. Bar, S. Shanmugaraju, K. Chib and P. S. Mukherjee, Dalton Trans., 2011, 40, 2257.

28 T. Shiraki, Y. Tsuchiya and S. Shinkai, Chem. Lett., 2010, 39, 156.

29 S. J. Toal, J. C. Sanchez, R. E. Dugan and W. C. Trogler, J. Forensic Sci., 2007, 52, 79.

30 Y. Liu, K. Ogawa and K. S. Schanze, J. Photochem. Photobiol., C, 2009, 10, 173.

31 P. M. Fierro-Mercado and S. P. Hernandez-Rivera, Int. J. Spectrosc., 2013, 2013, 1.

32 S. Z. Nergiz, N. Gandra, M. E. Farrell, L. Tian, P. M. Pellegrino and S. Singamaneni, J. Mater. Chem. A, 2013, 1, 6543. 